University of Nebraska - Lincoln

DigitalCommons@University of Nebraska - Lincoln

$2-2011$

\title{
Reversible collapse of the Langmuir films of a series of triphenylsilyl ether-terminated amphiphiles
}

\author{
Christine A. DeVries \\ University of Nebraska-Lincoln, christine.devries@wartburg.edu \\ James J. Haycraft \\ University of Nebraska-Lincoln \\ Qiang Han \\ University of Nebraska-Lincoln \\ Farhana Noor-e-Ain \\ University of Nebraska-Lincoln \\ Joseph Raible \\ University of Nebraska-Lincoln \\ See next page for additional authors
}

Follow this and additional works at: https://digitalcommons.unl.edu/chemistrydussault

Part of the Chemistry Commons

DeVries, Christine A.; Haycraft, James J.; Han, Qiang; Noor-e-Ain, Farhana; Raible, Joseph; Dussault, Patrick; and Eckhardt, Craig J., "Reversible collapse of the Langmuir films of a series of triphenylsilyl ether-terminated amphiphiles" (2011). Patrick Dussault Publications. 12.

https://digitalcommons.unl.edu/chemistrydussault/12

This Article is brought to you for free and open access by the Published Research - Department of Chemistry at DigitalCommons@University of Nebraska - Lincoln. It has been accepted for inclusion in Patrick Dussault Publications by an authorized administrator of DigitalCommons@University of Nebraska - Lincoln. 


\section{Authors}

Christine A. DeVries, James J. Haycraft, Qiang Han, Farhana Noor-e-Ain, Joseph Raible, Patrick Dussault, and Craig J. Eckhardt 


\title{
Reversible collapse of the Langmuir films of a series of triphenylsilyl ether-terminated amphiphiles
}

\author{
Christine A. DeVries, James J. Haycraft, Qiang Han, Farhana Noor-e-Ain, \\ Joseph Raible, Patrick H. Dussault, and Craig J. Eckhardt \\ Department of Chemistry and Nebraska Center for Materials and Nanoscience, \\ University of Nebraska-Lincoln, Lincoln, NE 68588-0304, USA \\ Corresponding author - C. J. Eckhardt, tel 402 472-2734, fax 402 472-9402, email eckhardt@unlserve.unl.edu \\ C. A. DeVries current address - Department of Chemistry and Engineering Science, \\ Wartburg College, Waverly, IA 50677, USA; christine.devries@wartburg.edu \\ J. J. Haycraft current address - Energetics Research Division (Code 474300D), \\ Naval Air Warfare Center Weapons Division, China Lake, CA 93555-6100, USA
}

\begin{abstract}
The Langmuir films of a series of triphenylsilyl ether (TPSE)-terminated amphiphiles were investigated in order to explore the bulge amphiphile model developed by Haycraft et al. [Thin Solid Films, 515 (2007) 2990]. The TPSE series was examined using surface pressure-surface area isotherms over a range of temperatures (287-298 K) with simultaneous acquisition of Brewster angle microscopy images. The Langmuir films of the TPSE series exhibit the reversible collapse process characteristic of bulge amphiphiles, including an observation consistent with the reversed cybotactic cluster model and increased entropy upon collapse. No solid-like monolayer phases were found over the temperature range studied. The reversed cybotactic clusters displayed for these films show domain growth and coalescence that differ from those observed by Haycraft et al.
\end{abstract}

Keywords: monolayers, bulge amphiphile, reversible collapse, reversed cybotactic clusters, brewster angle microscopy, Langmuir films

\section{Introduction}

Myriad amphiphiles, from small molecules to proteins, have been studied as monolayer films at the air-water interface. Traditional amphiphiles, such as fatty acids, have been examined with numerous techniques and are the paradigm for amphiphile film behavior [1, 2]. The primary model used to describe fatty acid Langmuir films is based on the similarities between monolayer phases and liquid crystals [3]. The liquid-crystal model assumes that Langmuir films form a hexatic arrangement. A model that does not require hexatic structures to describe Langmuir film phase behavior has been developed using a mean-field approach appropriate for the solid state [4].

Haycraft et al. studied benzo[c]phenanthrene-4-oxyundecanol (4BPO11), a surface-active molecule with an alcohol head group, a flexible hydrocarbon chain, and a fused-ring terminus (see Figure 1a) [5]. Haycraft et al. name this type of molecule a bulge amphiphile and suggest its Langmuir film properties best fit the liquid-crystal model because collapse into the third dimension is reversible, with Brewster angle microscopy (BAM) images displaying bright spots ascribed to reversed cybotactic clusters, structures similar to the cybotactic clusters found for some liquid crystals at the smectic-nematic transition [6-9]. This argument is used to define the 4BPO11 monolayer as smectic-like and the final collapsed structure as a more disordered nematic-like multilayer film [5].

The authors predict that the film behavior exhibited by
4BPO11 should be observed for any amphiphile containing a hydrophilic head, a flexible hydrophobic chain, and a stiff region of relatively large cross-sectional area compared to that of the chain. In the present study, the thin-film phase behavior of a series of bulge amphiphiles at the air-water interface is explored and compared to the Langmuir film phase behavior of 4BPO11 and other amphiphiles. These results help discriminate between general phenomena for the class of bulge amphiphiles and behavior specific to each molecule. In particular, the change in entropy associated with the monolayer collapse transition provides a test for the increased disorder expected for the reversible collapse mechanism proposed by Haycraft et al.

This study begins with 1,14-tetradecanediol, 14-triphenylsilyl ether (TPSE-C14), an amphiphile illustrated in Figure 1b. Because Langmuir films are studied on a water surface, there is a limit to the temperature range available for a given amphiphile. A common technique for exploring phenomena equivalent to low-temperature behavior is to extend the hydrocarbon chain. A series of $n$-alkanoic acids has been shown to display identical phase behaviors if the temperature is decreased by about $5 \mathrm{~K}$ for each added methylene unit [10]. Examining a series of amphiphiles with increasing chain length allows detection of low-temperature phenomena that are experimentally inaccessible for the TPSE-C14 Langmuir film. The series of amphiphiles examined in this research project are illustrated in Figure 2, with both the full chemical names and the abbreviated names used throughout this paper. 


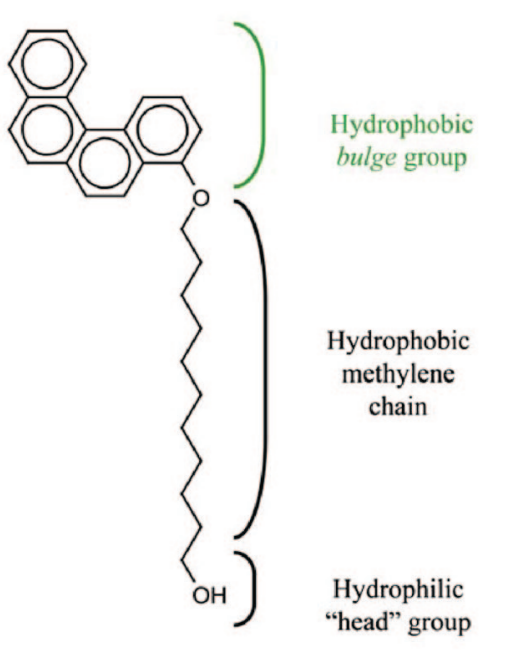

(a)

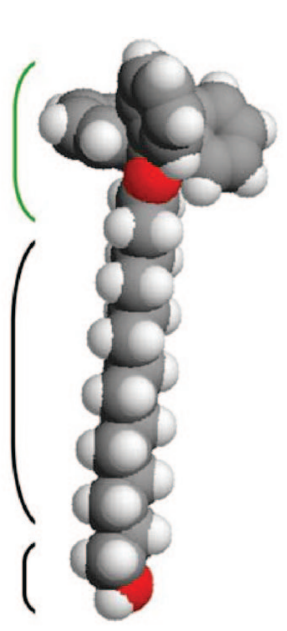

(b)

Figure 1. Illustrations of bulge amphiphiles (a) benzo[c]phenanthrene4-oxyundecanol (4BPO11) [5], and (b) space-filled model of TPSE-C14.

After relating experimental details in the next section, the results are presented and discussed. The findings of this work are then summarized in the conclusions section.

\section{Experimental details}

\subsection{Preparation of triphenylsilyl ethers TPSE-C14, TPSE-C16, and TPSE-C18}

Diol precursors: 1, 16-Hexadacanediol [CAS 7735-42-4] was used as received. 1, 14-Tetradecanediol [CAS 19812-64-7] was prepared via $\mathrm{LiAlH}_{4}$ reduction of either the commercially available tetradecanedioic acid or the corresponding dimethyl ester, which could be prepared in quantitative yield by refluxing a methanolic solution of the acid in the presence of a catalytic amount of toluenesulfonic acid. 1,18-Octadecanediol [CAS 3155-43-9] was prepared from octadecanedioic acid via formation of the dimethyl ester and reduction with $\mathrm{LiAlH}_{4}$.

Triphenylsilyl ethers: The triphenylsilyl ethers were prepared by addition of equimolar amounts of imidazole and triphenylsilyl chloride to DMF or ethyl acetate (hot) solutions of tetradecane-1,14-diol (TPSE-C14), hexadecane-1,16-diol (TPSE-C16), or octadecane-1,18-diol (TPSE-C18). Reactions were allowed to proceed for $24 \mathrm{~h}$, typically resulting in a mixture of the bissilyl ether, the desired monotriphenylsilyl ether, and recovered diol. The reactions were concentrated under vacuum and the residue was purified by chromatography on silica gel, using either $20 \%$ ethyl acetate/hexane or dichloromethane as the eluting solvent. In either solvent system, the bistriphenylsilyl ether byproducts elute first, followed by the desired monosilyl ether. The monosilyl ethers were colorless or light yellow waxy solids which gave expected ions by high-resolution mass spectrometry, and were judged to be of at least $98 \%$ purity based upon ${ }^{1} \mathrm{H}$ and ${ }^{13} \mathrm{C}$ nuclear magnetic resonance spectroscopy.

\subsection{Instrumentation}

The monolayer phase behaviors were identified through the standard isotherm method, measuring surface pressure as two barriers compressed or expanded the film at the air-water interface. Visual evidence of the Langmuir film phase behavior was obtained through Brewster angle microscopy with a HeNe laser, polarizer, focusing lens, and CCD digital video camera. The experimental instrumentation used was the same

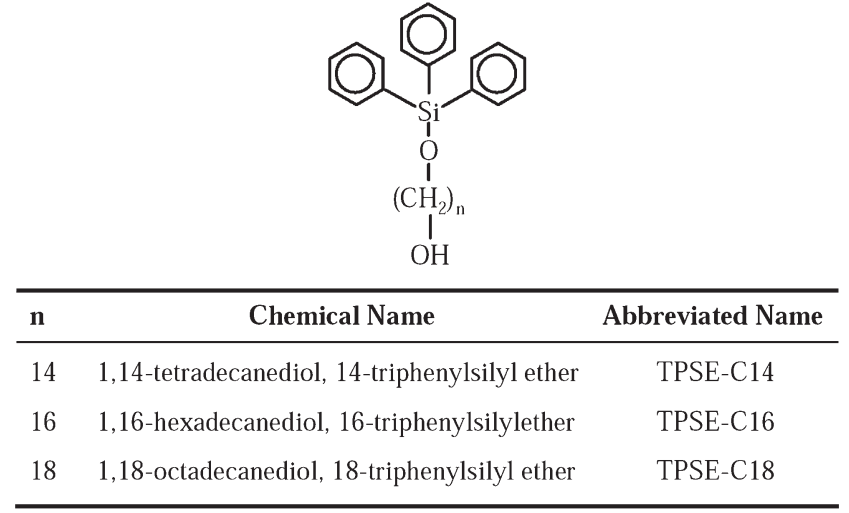

Figure 2. Illustration indicating the amphiphiles examined in this article.

as previously described [5], with minor changes: a Millipore Direct Q-3 system was used to obtain ultrapure water (18.2 $\mathrm{M} \Omega \cdot \mathrm{cm}$ resistivity); BAM images captured on VHS tapes were transferred to digital media through a Canon ZR200 Digital Video Camera; and individual frames were subsequently obtained with the iMovie program (Apple Computers) and individually processed with Adobe Photoshop Elements 5.0 software to adjust brightness and contrast to improve discrimination of features.

The molecular model illustrations in Figure 1 and Figure 2 were prepared with ACD/ChemSketch Freeware.

\subsection{Monolayer preparation}

Amphiphile solutions (0.1-0.8 mg/mL) were prepared using Optima grade chloroform (Fisher Scientific). The solution was spread dropwise upon the water surface from a 50, 100, or $250 \mu \mathrm{L}$ Hamilton microsyringe. The system was allowed to rest for 30-180 min to allow the solvent to evaporate. The annealing process consisted of several compression/expansion cycles to low surface pressure $(\sim 1 \mathrm{mN} / \mathrm{m})$, and then to subsequently increasing surface pressures $(\sim 10 \mathrm{mN} / \mathrm{m})$. To keep close to equilibrium, relatively slow compression rates (4-8 $\AA^{2} /$ molecule $\cdot$ min) were maintained.

\section{Results and discussion}

The amphiphiles of interest were studied as insoluble monolayers at the air-water interface. Isotherms below, above, and at room temperature $(293 \mathrm{~K})$ were obtained for each of the TPSE-terminated amphiphiles, to observe the phase behavior for these Langmuir films. BAM images were used to confirm the phase changes and to gain insight into the behavior of the amphiphiles in Langmuir film.

\section{1. $\pi-a$ isotherms of the TPSE series}

A typical room temperature $(293 \mathrm{~K})$ surface isotherm for TPSE-C14 is shown in Figure 3. Two characteristic points may be identified for a single compression-expansion cycle. Starting at maximum area (> $50 \AA^{2} /$ molecule) with the compression portion of an isotherm cycle, the isotherm is essentially flat at low surface pressure. This isotherm behavior is characteristic of the gas-analogue monolayer phase [1]. As the surface area approaches $40 \AA^{2} /$ molecule, the surface pressure abruptly increases. The increased magnitude of the slope in the isotherm indicates a more condensed monolayer phase is present, such as the liquid- or solid-type phase. The steepest part of the curve is fit with a linear regression. The $x$-intercept of this line gives the characteristic parameter $\mathrm{A}_{0}$, the molecular area of the phase extrapolated to zero surface pressure. The average $A_{0}$ values for 


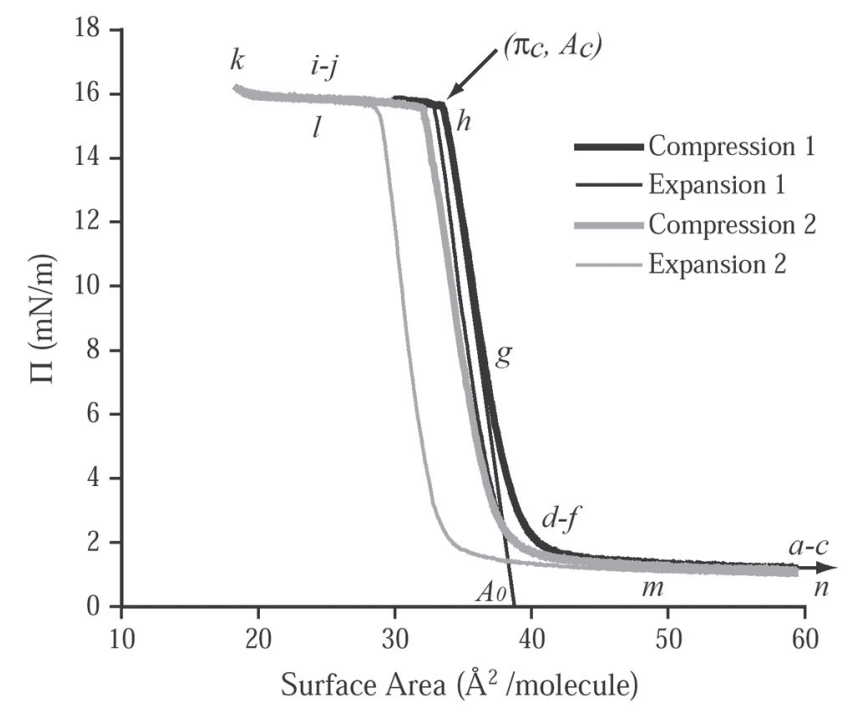

Figure 3. Two consecutive isotherms of TPSE-C14 at $293 \mathrm{~K}$.

TPSE-C14 at four temperatures are listed in Table 1. No temperature-dependent trend is observed, which implies that the molecular cross-sectional area of this phase is consistent regardless of temperature. Any hysteresis during successive compressionexpansion cycles will affect the value observed for $A_{0}$. It is this small permanent surface area loss among repeated isotherm measurements that accounts for the rather large standard deviations in these area measurements.

A conspicuous transition occurs with continued compression: a sudden shift in slope to a nearly horizontal pla teau (see Figure 3 ). The range of this plateau begins near the estimated minimum area before collapse, $38.0 \AA^{2} /$ molecule [11], and is observed to continue to the alkanoic acid collapse transition area, $\sim 20 \AA^{2} /$ molecule [12]. The area, which is less than the calculated cross-section of the amphiphile, implies transition to a three-dimensional structure. The collapse surface pressure $\left(\Pi_{c}\right)$ is highly reproducible among multiple isotherms for the same amphiphile, while the surface area of the collapse transition $\left(A_{c}\right)$ is affected by a small hysteresis among subsequent isotherm cycles, again leading to rather large standard deviations in the area measurements. The average values for the collapse transition surface area and surface pressure for TPSE$\mathrm{C} 14$ at four temperatures are listed in Table 1. Again, there is no temperature-dependent trend for the characteristic surface area $\left(A_{c}\right)$, but a small temperature dependence is noted for the collapse surface pressure $\left(\Pi_{\mathrm{c}}\right)$. This trend is discussed below.

Expansion of the compressed films derived from TPSE-14 reveals markedly different behaviors compared with films derived from collapsed fatty acid monolayers. Rather than the typical sudden drop in surface pressure indicating an irreversible

Table 1. Characteristic points for TPSE-C14, TPSE-C16, and TPSE-C18.

\begin{tabular}{lllll}
\hline Amphiphile & $\begin{array}{l}\text { Temperature } \\
(\mathrm{K})\end{array}$ & $\begin{array}{l}\mathrm{A}_{0} \\
\left(\AA^{2} / \text { molecule }\right)\end{array}$ & $\begin{array}{l}\mathrm{A}_{c} \\
\left(\AA^{2} / \text { molecule }\right)\end{array}$ & $\begin{array}{l}\Pi_{\mathrm{c}} \\
(\mathrm{mN} / \mathrm{m})\end{array}$ \\
\hline TPSE-C14 & 296 & $37.9 \pm 6.1$ & $30.5 \pm 5.9$ & $14.5 \pm 0.6$ \\
& 293 & $39.8 \pm 2.6$ & $32.2 \pm 2.9$ & $15.0 \pm 0.3$ \\
& 289 & $37.9 \pm 2.7$ & $30.1 \pm 2.0$ & $15.2 \pm 0.1$ \\
TPSE-C16 & 287 & $42.3 \pm 2.4$ & $36.6 \pm 1.2$ & $15.5 \pm 0.5$ \\
& 296 & $35.2 \pm 3.7$ & $30.5 \pm 1.9$ & $14.9 \pm 0.4$ \\
& 293 & $37.1 \pm 1.8$ & $28.9 \pm 2.7$ & $15.1 \pm 0.3$ \\
& 289 & $36.7 \pm 0.1$ & $30.5 \pm 0.7$ & $16.0 \pm 0.2$ \\
TPSE-C18 & 287 & $38.2 \pm 2.1$ & $31.1 \pm 1.9$ & $16.0 \pm 0.1$ \\
& 298 & $32.2 \pm 2.7$ & $23.0 \pm 1.9$ & $15.6 \pm 0.2$ \\
& 293 & $27.2 \pm 3.3$ & $20.8 \pm 2.8$ & $16.0 \pm 0.5$ \\
& 289 & $32.7 \pm 2.6$ & $25.3 \pm 1.9$ & $16.2 \pm 0.3$ \\
& 287 & $32.9 \pm 2.1$ & $25.3 \pm 2.2$ & $16.4 \pm 0.2$ \\
\hline
\end{tabular}

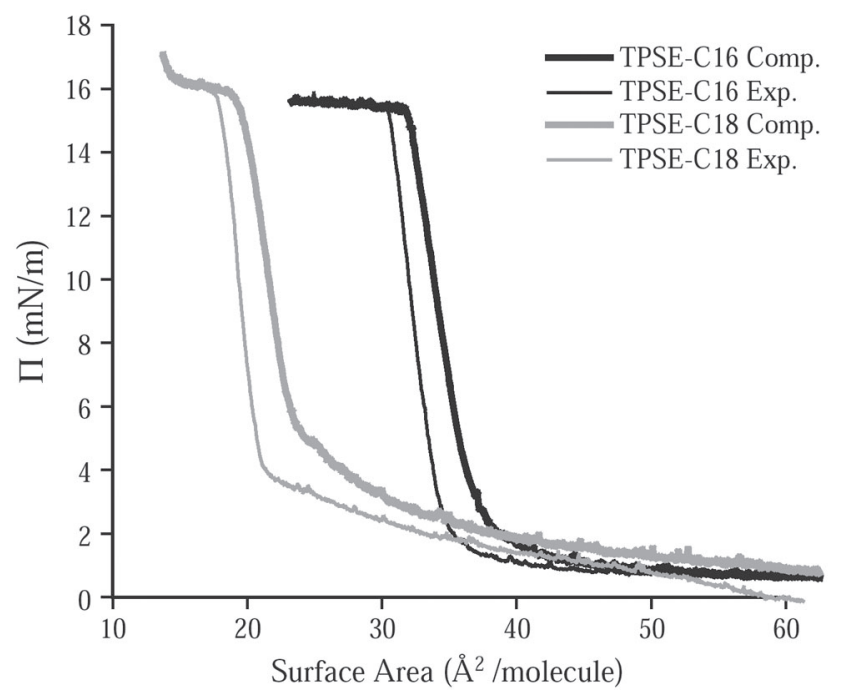

Figure 4. Isotherms of TPSE-C16 (black) and TPSE-C18 (gray) at $293 \mathrm{~K}$.

collapse process, the TPSE-C14 expansion curve essentially retraces the compression curve, a behavior similar to that previously reported for bulge amphiphile films. Figure 3 shows the expansion curve retracing the compression curve along the collapse transition plateau with a drop in surface pressure just before returning to the collapse transition point area. Not only do the TPSE series amphiphiles display reversibility in a single isotherm, but consecutive compression-expansion cycles retrace the original isotherm even after moderate compression beyond $A_{c}$. The area difference between the first compression and first expansion curves appears to be a small permanent loss in area, since the second compression nearly traces the first expansion curve, as illustrated in Figure 3.

The $293 \mathrm{~K}$ isotherms for TPSE-C16 and TPSE-C18 are nearly identical to that of TPSE-C14, as shown in Figure 4. The surface area at zero pressure $\left(A_{0}\right)$ and the collapse area $\left(A_{c}\right)$ for TPSE-C18 fall at smaller areas than the corresponding points for TPSE-C14 and TPSE-C16 films (see Table 1). One may expect identical cross-sectional areas $\left(\mathrm{A}_{0}\right)$ for the three molecules, but experiments showed slightly more hystereses during annealing with TPSE-C18, thus giving smaller $A_{0}$ and $A_{c}$ values for the longest molecule. Each individual amphiphile shows no temperature-dependent trend in these characteristic areas; these values simply have some variability due to the accumulation of hysteresis among anneals and consecutive isotherms. The collapse surface pressure $\left(\Pi_{c}\right)$ is observed at higher surface pressures as the molecular length is increased, which is consistent with treating the isotherms of longer molecules as corresponding to lower temperatures that are experimentally inaccessible for TPSE-C14. This temperature dependence is consistent with that observed for each molecule: the collapse surface pressure increases with decreasing temperature. For all three TPSE-terminated amphiphiles, both the expansion and subsequent compression show negligible hystereses.

\subsection{Monolayer compressibilities}

The two-dimensional compressibility $\left(\mathrm{C}_{\mathrm{m}}\right)$ is given by:

$$
\mathrm{C}_{\mathrm{m}}=\frac{1}{A} \cdot\left(\frac{\partial A}{\partial \pi}\right)_{T}
$$

where $A$ is the molecular area and $(\partial A / \partial \pi)_{T}$ is the inverse of the slope of the isotherm through the given phase [1]. The slope for the condensed phase is calculated using a linear regression of the curve in that region. Each regression employs $30-50$ points along the straightest part of the curve giving an 
$\mathrm{R}^{2}$ value greater than 0.99 . The condensed molecular area $(A)$ was determined [11] to be $38.0 \AA^{2} /$ molecule. The compressibilities were calculated from several isotherm experiments for each amphiphile (TPSE-C14, TPSE-C16, and TPSE-C18); average values are listed in Table 2 . The literature values observed for stearic acid [12] and octadecanol [13] are also included in Table 2 for comparison. From these data, the more compressible nature of condensed TPSE-C14, TPSE-C16, and TPSEC18 monolayers is evident compared to the solid-like (LS, S) phases of $n$-alkanoic acids or alcohols.

\subsection{Temperature studies}

Some amphiphiles exhibit monolayer phases that are only accessible with temperature studies. A transition point or plateau may appear or disappear at temperatures greater or less than room temperature. Since the TPSE-C14, TPSE-C16, and TPSE-C18 Langmuir films do not show any significant changes in the general shape of their isotherms with temperature, no explicit temperature-induced phase changes in these materials is found in the temperature range of the measurements. The characteristic surface areas $\left(A_{0}, A_{c}\right)$ show no temperature-dependent trend, nor do the monolayer compressibilities of the condensed monolayer phase. However, the temperature studies do reveal that the collapse surface pressure increases with decreasing temperature, as indicated in Table 1 . This trend is essential in determining the change in entropy over the reversible collapse transition.

\subsection{Entropy of the reversible collapse transition}

A first-order phase transition is identified by an abrupt change of slope in the isotherm followed by a plateau of nearly constant surface pressure over decreasing area [14]. The plateau is a region of coexistence when two phases of the same material are in dynamic equilibrium. As the more expanded initial phase is compressed, an increasing proportion of the more compressed phase appears. The first-order transition coexistence plateau has been observed between two monolayer phases [15] and between a monolayer and its collapsed threedimensional structure [16].

The entropy change of the phase transition associated with the main collapse plateau can be explored qualitatively with the data presented above. For a first-order phase transition in a given monolayer, the Clausius-Clapeyron equation may be written as:

$$
\frac{\Delta H}{T}=\Delta S=\frac{d \pi}{d T} \Delta A
$$

Table 2. Compressional monolayer compressibilities for octadecanoic (stearic) acid, octadecanol, TPSE-C14, TPSE-C16, and TPSE-C18.

\begin{tabular}{|c|c|c|c|}
\hline \multicolumn{2}{|l|}{ Amphiphile (phase) } & $\begin{array}{l}\text { Temperature } \\
(\mathrm{K})\end{array}$ & $\begin{array}{c}\mathrm{C}_{\mathrm{m}} \\
\left(\times 10^{-3} \mathrm{~m} / \mathrm{mN}\right)\end{array}$ \\
\hline \multirow[t]{4}{*}{ TPSE-C14 } & & 296 & $11.3 \pm 1.3$ \\
\hline & & 293 & $9.9 \pm 1.4$ \\
\hline & & 289 & $10.4 \pm 0.7$ \\
\hline & & 287 & $9.7 \pm 1.0$ \\
\hline \multirow[t]{4}{*}{ TPSE-C16 } & & 296 & $10.7 \pm 1.3$ \\
\hline & & 293 & $11.2 \pm 3.0$ \\
\hline & & 289 & $8.7 \pm 1.3$ \\
\hline & & 287 & $10.1 \pm 1.4$ \\
\hline \multirow[t]{4}{*}{ TPSE-C18 } & & 298 & $13.3 \pm 1.0$ \\
\hline & & 293 & $9.8 \pm 1.2$ \\
\hline & & 289 & $10.7 \pm 2.1$ \\
\hline & & 287 & $8.9 \pm 1.2$ \\
\hline \multirow{2}{*}{ Octadecanoic acid [12] } & Liquid-like & 293 & $9.05 \pm 0.23$ \\
\hline & Solid-like & & $0.98 \pm 0.04$ \\
\hline \multirow[t]{3}{*}{ Octadecanol [13] } & $\mathrm{L}_{2}$ & 298 & $6.8 \pm 1.3$ \\
\hline & LS & & $1.6 \pm 0.2$ \\
\hline & S & 280 & $0.7 \pm 0.1$ \\
\hline
\end{tabular}

where $H$ is the enthalpy, $T$ is temperature, $S$ is entropy, $\pi$ is surface pressure, and $A$ is the surface area. Defining the larger area phase as initial, and the more compressed phase as final, gives a negative value for $\Delta A$, the width of the phase transition plateau. Measuring isotherms at several temperatures allowed evaluation of the plateau's shift in surface pressure with temperature. The collapse transition plateau shifts to lower surface pressure with increasing temperature, making $(d \pi / d T)$ negative. Overall, this indicates a positive value for $\Delta S$ for the transition from the expanded to the condensed phase.

Pallas and Pethica observed a small decrease in entropy over the liquid-expanded to liquid-condensed transition for a Langmuir film prepared from $n$-pentadecanoic acid [17], presumably resulting from increased molecular ordering with condensation. Bommarito et al. [18] calculated the entropy changes for the $\mathrm{L}_{2}{ }^{\prime} \rightarrow \mathrm{L}_{2}{ }^{\prime \prime}$ and $\mathrm{L}_{2} \rightarrow \mathrm{L}_{2}$ " transitions of behenic acid $\left(\mathrm{CH}_{3}\left(\mathrm{CH}_{2}\right)_{20} \mathrm{COOH}\right)$. Both of these transitions may be observed by decreasing temperature or increasing surface pressure. Bommarito et al. attribute the negative entropy changes as due to the first-order crystallization of a less-ordered phase to a well-ordered, crystalline phase. In contrast, amphiphiles with a larger cross-section, including glycolipids and phospholipids, have shown an increase in entropy as the Langmuir film is compressed from the liquid-expanded to the liquidcondensed phase [19].

Sigl et al. [20] examined the monolayer to bilayer-multilayer transition for Langmuir films of two different "hairy rod" polymers, polymers containing a non-amphihilic rod-shaped core which is substituted with alkane side-chains. The authors observed that the collapse transition surface pressure of films prepared from the polymer decreases with increasing temperature, implying a positive entropy change with compression. Ibn-Elhaj et al. have observed a small entropy increase for the monolayerto-multilayer transition for films derived from three-block organosilane amphiphiles $5 \mathrm{AB}_{2}$ and $5 \mathrm{AB}_{3}$. They ascribe the small entropy increase to a change in water ordering [21]. Plehnert et al. have examined a series of mesogenic amphiphiles in which the liquid crystalline and Langmuir film ordering are in competition. These systems show a positive change in entropy exhibited upon collapse. The authors attribute the entropy increase to two driving factors: the shift from "a polar to an unpolar ordering" (from a monolayer at the air-water interface to a centrosymmetric liquid crystalline double layer on top of the monolayer), and the reordering of water [22].

Much like the work summarized above, the entropy increase with collapse observed for the TPSE series Langmuir films may be due to water and/or amphiphile reordering. A surface-sensitive optical-microscopy technique was used to obtain additional evidence of amphiphile reordering upon the collapse transition.

\subsection{Brewster angle microscopy}

Brewster angle microscopy (BAM) [23, 24] was employed for real-time visual observation of the Langmuir films throughout the various experiments. The darkest BAM images represent regions of pure water, while the brightest images represent regions with a highest density of organic material. Figure 5 displays BAM images of the TPSE-C14 film at a series of points along the isotherms, as described below. The BAM images were selected from experiments at various temperatures to illustrate the consistent, temperature-independent phase behavior of the TPSE-C14 film. The corresponding location of each BAM image along an isotherm is indicated with lower-case letters in Figure 3. The BAM images of the TPSE-C16 and TPSE-C18 films are essentially identical and are not included here.

After spreading the amphiphile solution over the surface of the water subphase and allowing time for the solvent to evaporate, the Langmuir film exhibits large regions of darker and lighter domains flowing past one another. After annealing, 


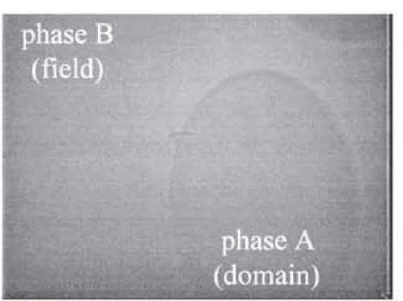

(a) $14^{\circ} \mathrm{C}, 112 \AA^{2} /$ molecule, $\sim 0 \mathrm{mN} / \mathrm{m}$

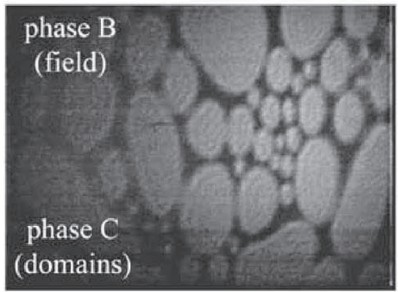

(d) $23^{\circ} \mathrm{C}, 35 \AA^{2} /$ molecule, $1.8 \mathrm{mN} / \mathrm{m}$

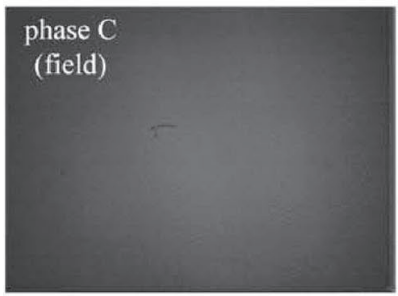

(g) $16^{\circ} \mathrm{C}, 35 \AA^{2} /$ molecule, $8.0 \mathrm{mN} / \mathrm{m}$

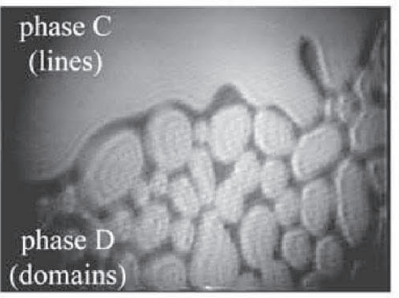

(j) $20^{\circ} \mathrm{C}, 22.5 \AA^{2} /$ molecule, $15.3 \mathrm{mN} / \mathrm{m}$

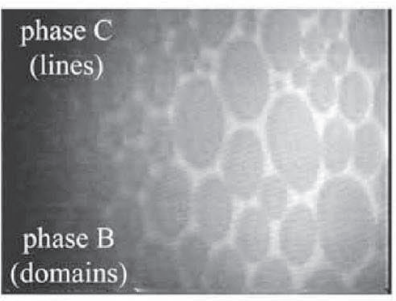

(m) $20^{\circ} \mathrm{C}, 47 \AA^{2} /$ molecule, $0.5 \mathrm{mN} / \mathrm{m}$

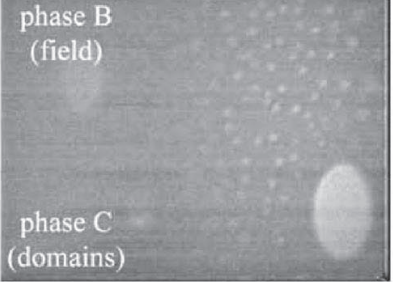

(b) $23^{\circ} \mathrm{C}, 70 \AA^{2} /$ molecule, $0.5 \mathrm{mN} / \mathrm{m}$

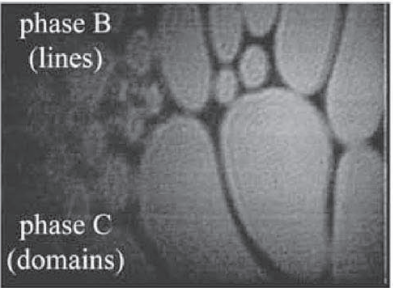

(e) $23^{\circ} \mathrm{C}, 35 \AA^{2} /$ molecule, $1.8 \mathrm{mN} / \mathrm{m}$

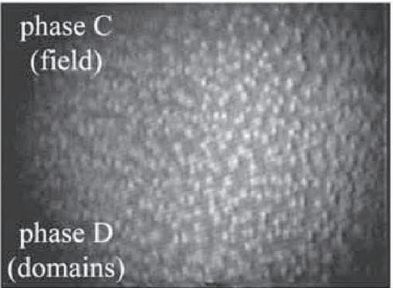

(h) $20^{\circ} \mathrm{C}, 25 \AA^{2} /$ molecule, $15 \mathrm{mN} / \mathrm{m}$

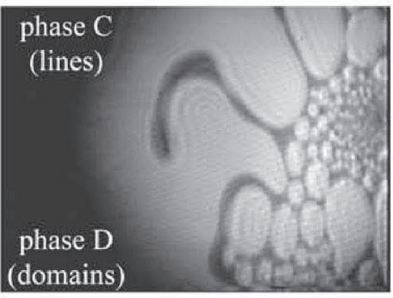

(K) $20^{\circ} \mathrm{C}, 18 \AA^{2} /$ molecule, $15.8 \mathrm{mN} / \mathrm{m}$

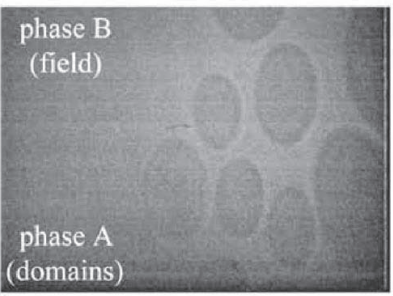

(n) $14^{\circ} \mathrm{C}, 103 \AA^{2} /$ molecule, $\sim 0 \mathrm{mN} / \mathrm{m}$

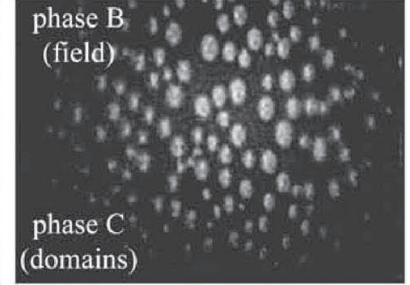

(C) $14^{\circ} \mathrm{C}, 63 \AA^{2} /$ molecule, $0.9 \mathrm{mN} / \mathrm{m}$

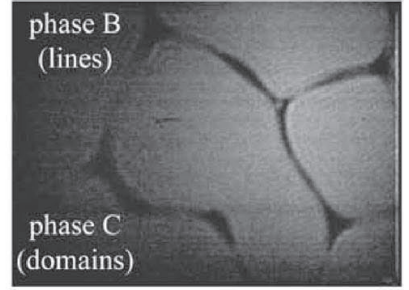

(f) $23^{\circ} \mathrm{C}, 35 \AA^{2} /$ molecule, $1.8 \mathrm{mN} / \mathrm{m}$

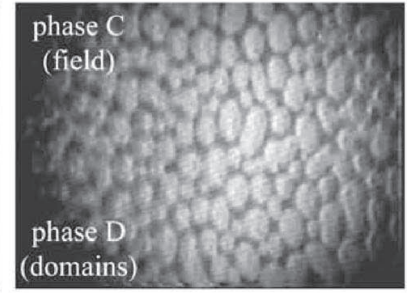

(i) $20{ }^{\circ} \mathrm{C}, 22.5 \AA^{2} /$ molecule, $15.3 \mathrm{mN} / \mathrm{m}$

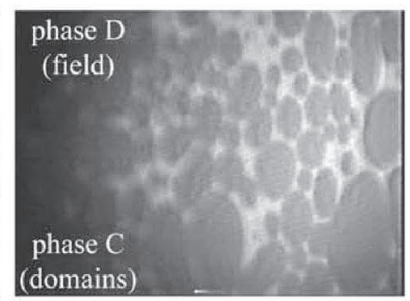

(1) $20^{\circ} \mathrm{C}, 24.5 \AA^{2} /$ molecule, $14.5 \mathrm{mN} / \mathrm{m}$

Figure 5. a-m: Brewster angle microscopy (BAM) images of TPSE-C14, $\sim 1 \mathrm{~mm}^{2}$.

these coexisting phases continue to be observed, as illustrated in Figure 5a. The darkest phase, indicative of little or no organic material, is labeled phase A for ease of discussion. The brighter phase in these initial images is referred to as phase $B$.

With continued compression, the phase B domains grow and coalesce into a homogenous layer. Figure $5 \mathrm{~b}$ shows the homogenous phase B film interrupted with small, bright dots (phase C). Over the next $\sim 40 \AA^{2} /$ molecule of compression, these bright dots grow in number and size, as indicated in Figure $5 \mathrm{c}$. As the surface pressure begins to rise for the main condensed phase, these regions coalesce (see Figure $5 \mathrm{~d}-\mathrm{f}$ ) until a homogenous film of phase $\mathrm{C}$ is observed (Figure $5 \mathrm{~g}$ ). This fea- tureless image is observed as surface pressure increases until the collapse transition point is reached. In the plateau just beyond the collapse transition point, very bright, approximately circular dots appear (Figure 5h). Compression along this plateau increases the number and size of these phase D dots, with coalescence as shown in Figure 5i-k. This collapse behavior differs from that observed by Haycraft et al. for 4BPO11 Langmuir films. In that case, the bright spots present over the collapse plateau neither grow in size nor coalesce upon compression.

Upon expansion, the BAM images of the film appear similar to the compression images. The phase $\mathrm{D}$ layer converts to phase 
C (Figure 51), phase C converts to phase B (Figure $5 \mathrm{~m}$ ), and at very large molecular areas, coexisting phases A and B are observed (Figure 5n). The primary difference between the compression and expansion images is the shape of each phase during coexistence (compare Figure $5 \mathrm{j}$ and 1 or Figure $5 \mathrm{~d}$ and $\mathrm{m}$ ). In both directions, the growing phase is more or less circular and exhibits coalescence, while the waning phase forms a foam-like structure that withdraws along the isotherm. Foam-like structures have previously been observed for Langmuir films of other amphiphiles, such as 4'-n-octyl-4-cyanobiphenyl upon expansion [25], stearic acid in the liquid-gas coexistence region [26], and poly(dimethylsiloxane) in the submonolayer regime [27].

To further examine the phase behavior of these Langmuir films, a polarizing film was placed between the lens and detector to detect the presence and relative direction of molecular tilt $[25,28]$. Examination of films prepared from the three individual amphiphiles showed no regions of differing brightness within or among the phases, thereby giving no measurable indication of collective molecular tilt. The apparent lack of molecular tilt differentiates the TPSE series from traditional amphiphiles, and this structural feature must be incorporated into the model of the bulge amphiphile-monolayer phase behavior and reversible collapse mechanism.

\section{Discussion}

\subsection{TPSE series isotherms and BAM images}

The isotherms displayed above for the TPSE series of amphiphiles show very little hysteresis, either within individual compression-expansion cycles or among consecutive cycles. The comparison of estimated molecular cross-sectional area with the transition areas $\left(A_{c}\right)$, given in Table 1 , showed that the main plateau is associated with monolayer collapse into the third dimension. Although most amphiphiles show reversibility up to the collapse point, typical amphiphile Langmuir films exhibit irreversible collapse mechanisms. The low hysteresis of the TPSE series isotherm curves indicates a reversible monolayer collapse mechanism. The BAM images support this conclusion, displaying similarity among compression and expansion images and identical BAM images with subsequent isothermal cycles. Such reversible collapse Langmuir film behavior is similar to that observed by Rolandi et al. [29] for a glycerol diether as well as Haycraft et al. [5] for the bulge amphiphile 4BPO11. Overall, the isotherms and BAM images demonstrate that the TPSE series (and bulge amphiphiles in general) exhibit a reversible collapse mechanism. The presence of the bulge group appears to prevent the solid state behavior exhibited by $n$-alkanoic acid or alcohol films.

The monolayer compressibilities calculated for the main surface pressure rise in the TPSE series isotherms can be compared to literature values for typical amphiphiles (see Table 2). The main surface pressure rise has the largest magnitude slope and, therefore, the smallest compressibility values. The TPSE series Langmuir films give monolayer compressibilities an order of magnitude larger than the liquid-condensed and solid-like monolayer phases of typical amphiphiles. Rather, the TPSE series monolayer compressibilities are comparable to the liquid-expanded monolayer phases in stearic acid or octadecanol films. The isotherms for the TPSE series exhibit similar high monolayer compressibilities even below room temperature that imply relatively fluid Langmuir films.

Oleic acid, a stearic acid analogue with a cis double bond in the middle of the methylene chain, is known to only form liquid-expanded phases at all experimentally accessible temperatures [1]. Ibn-Elhaj et al. have reported another amphiphile that does not form crystalline monolayer phases at the air-water interface [21]. Both of these amphiphiles are liquids at room temperature, which reflects the low intermolecular forces within these materials. The lack of strong intermolec- ular forces prevents both bulk and monolayer crystallization. In contrast, all three TPSE-terminated amphiphiles are waxy solids at room temperature (melting points for the three molecules range from $311 \mathrm{~K}$ to $321 \mathrm{~K}$ ). However, it is notable that the Langmuir film phase behavior for the TPSE series amphiphiles reflects lack of strong cohesive forces within a monolayer. The molecules do not form crystalline monolayers with compression, even at low temperatures. Also, real-time viewing of the BAM images of the TPSE series films display domains (phases A-D) flowing past each other and coalescing with compression, leaving no question that these are fluid films. The monolayer compressibilities and correlating BAM results show that the TPSE series exhibit no solid-like monolayer phases at experimentally accessible temperatures despite the high-melting solid phases of the bulk materials.

\subsection{Entropy of the reversible collapse transition}

The Langmuir films of TPSE-C14, TPSE-C16, and TPSE-C18 show entropy increases upon compression to the main collapse transition plateau. The reversible collapse mechanism describing the TPSE series, therefore, must include a description of amphiphile rearrangement to account for the increase in entropy with compression. This result is in agreement with the bulge amphiphile collapse mechanism proposed by Haycraft et al. [5] in which they suggest an increase in disorder as the film is compressed from the close-packed monolayer to the three-dimensional post-collapse structure, as summarized below.

\subsection{Bulge amphiphile reversible collapse mechanism}

Haycraft et al. describe the reversible collapse mechanism for bulge amphiphile Langmuir films in detail [5], so it is merely summarized here in light of the results from the TPSE series of bulge amphiphiles. The bulge amphiphile Langmuir film is treated in terms of sublayers, as suggested by Ibn-Elhaj et al. [21]. The main rise in surface pressure coincides with the bulge group sublayer reaching a closely packed arrangement near $38 \AA^{2} /$ molecule. At this surface area, the hydrocarbon-chain sublayer is not close-packed, rather there is substantial free volume between the chains due to the lollipop-like shape of the molecule, as indicated in Figure 6a. The bulge group prevents a close-packed arrangement of the hydrocarbon chains as the monolayer is compressed. However, with sufficient compression, a given molecule may shift up or down a fraction of its length out of the monolayer plane, as shown in Figure 6b. In this way, there is a relief of the stress. The lateral view of this post-collapse arrangement given in Figure $6 \mathrm{c}$ is similar to nematic regions growing within a smectic layer. This proposed coexistence of smectic and nematic phases is reminiscent of the cybotactic clusters observed in liquid crystals. Cybotactic clusters are domains of smectic mesophases contained within the field of a nematic mesophase and are found when a nematic liquid crystal is cooled to just above the smectic-nematic transition temperature $\left(\mathrm{T}_{\mathrm{AN}}\right)[6,9]$. The proposed bulge amphiphile Langmuir film collapse structure consists of growing nematic domains within a smectic layer, therefore, this inverted arrangement has been termed reversed cybotactic clusters [5].

The TPSE series of Langmuir films display BAM images along the collapse plateau that correspond with the proposed reversed cybotactic clusters: bright spots begin to appear just beyond the collapse point. Cybotactic clusters in liquid crystals are known to be a transitional phenomenon, with smectic clusters growing in size as the temperature decreases below $\mathrm{T}_{\mathrm{AN}}$, the smectic A-nematic transition temperature [30]. For the bulge amphiphiles, the nematic domains may be expected to grow within the smectic monolayer as the barriers are compressed. The very bright spots discovered in the BAM images of 4BPO11 films never appear to grow in size or coalesce [5]. In 

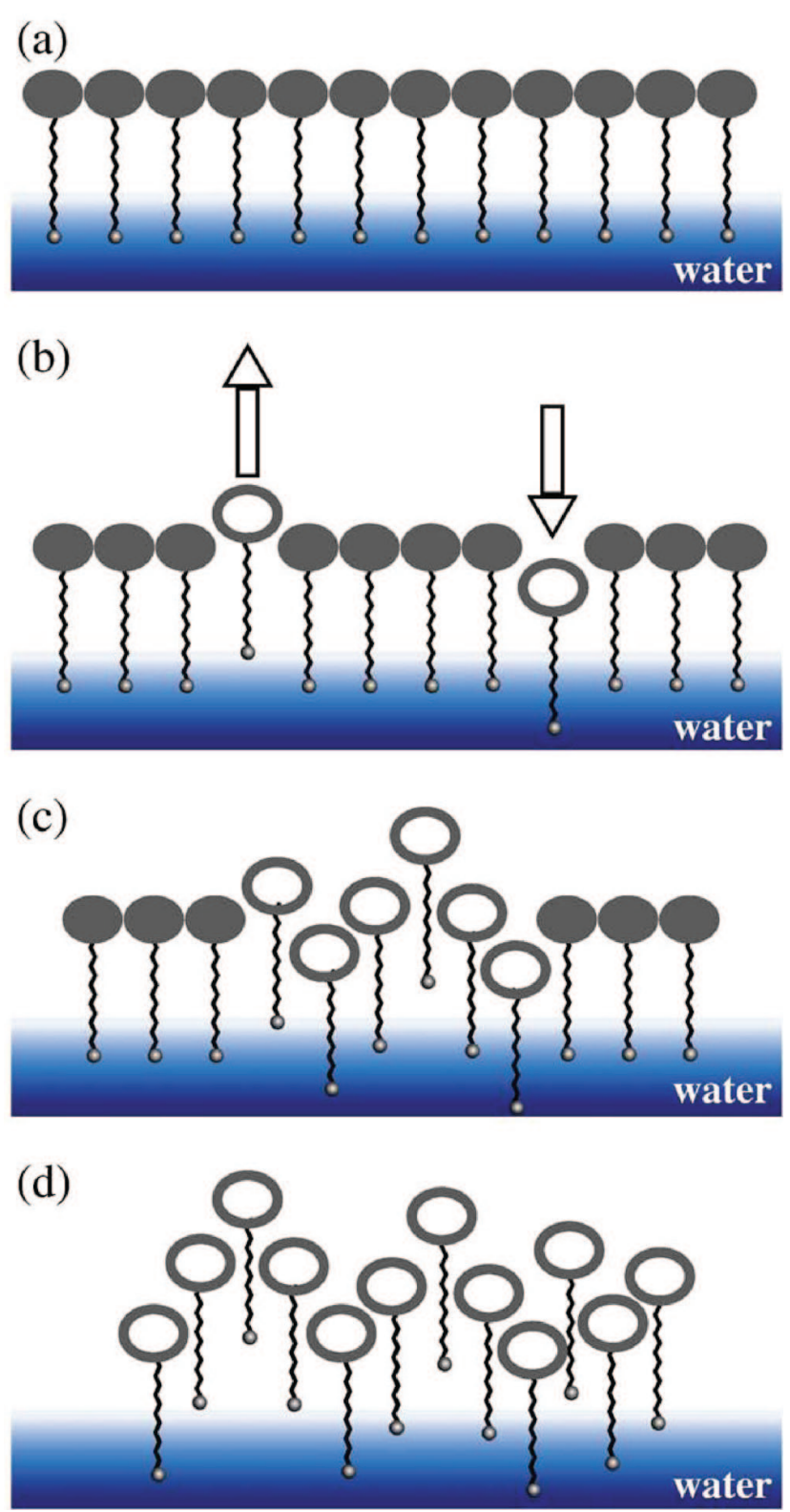

Figure 6. Bulge amphiphile reversible collapse mechanism (lateral view). (a) Smectic monolayer before collapse (phase C), (b) individual molecules shift out of sublayer (arrows), (c) reversed cybotactic clusters (nematic-like in smectic-like) and (d) collapsed nematic phase (phase D). Figure adapted from Haycraft et al. [5].

contrast, TPSE-C14, TPSE-C16 and TPSE-C18 Langmuir films exhibit post-collapse BAM images that follow the expectations for reversed cybotactic clusters. Phase $\mathrm{C}$, the smooth monolayer observed at the main rise in surface pressure, can be assigned as a smectic phase. Phase D, composed of bright domains that grow and coalesce upon compression, is observed over the post-collapse plateau (see Figure $5 \mathrm{~h}-\mathrm{k}$ ). The phase D domain behavior is in good agreement with the expectations for reversed cybotactic clusters. This conclusion implies that regular cybotactic clusters (smectic domains in a nematic field) may be observed on the transition from phase $D$ to phase $C$, as the barriers expand along the main plateau (see Figure 5l).

\subsection{Model to describe Langmuir films of bulge amphiphiles}

The liquid-crystal (LC) model for Langmuir films describes monolayers at the air-water interface in terms of the various smectic mesophases [3]. The LC approach supports the assignment of phase $\mathrm{C}$ as a smectic monolayer. Although a full theoretical development is not appropriate here, some observations can be made. The LC model of Langmuir films does not currently include an order parameter explicitly defined to describe a nematic phase with molecular excursions perpendicular to the interface (up and down). Because the LC model is composed of defined order parameters, rather than derived from first principles, there is no restriction to adding a new order parameter to describe the smectic-nematic transition. Following Kaganer's technique, an amplitude and an azimuth could be defined to describe the break in lamellar ordering. Alternatively, the smectic-nematic transition could be treated as an extension of the hexatic ordering parameter since this parameter does not currently describe any Langmuir film phase transitions.

The expansion of the LC model of Langmuir films to include a nematic phase (even though multilayered) would increase the parallels between two- and three-dimensional systems. One might expect the couplings between the "cybotactic" order parameter (or the extended hexatic) and the other four order parameters to play a significant role in the free energy expansion to describe the smectic-nematic transition. For example, one would expect the free energy change over the transition to depend upon the angle between the director and the normal to the interface for both the smectic and nematic phases. Using this more general approach, the liquidcrystal model may be applied to the bulge amphiphiles and any other "nematic" Langmuir films.

\section{Conclusions}

The goal of this research was to determine whether the Langmuir film behavior exhibited by $4 \mathrm{BPO} 11$ is general for bulge amphiphiles, as predicted by Haycraft et al. [5]. The preceding discussion has led to three conclusions about the TPSE series examined here. Langmuir films of the TPSE series exhibit: (1) no solid-like monolayer phase at experimentally accessible temperatures, despite the high-melting solid phases of the non-solvated bulk amphiphiles, (2) a reversible collapse mechanism that accounts for the increase in entropy and is consistent with formation of cybotactic clusters, and (3) phase behavior consistent with a modified liquid-crystal model.

These conclusions, along with the proposed reversible collapse mechanism, form the general understanding for the Langmuir film behavior of bulge amphiphiles. The relatively weak intermolecular forces driving the Langmuir film behavior are a result of the free volume available for each molecule due to their lollipop-like structure. Overall, these results confirm the bulge amphiphile Langmuir film behavior observed by Haycraft et al. [5] for 4BPO11, with a few notable additions. For the TPSE series, phase $\mathrm{D}$ domains within a field of phase $\mathrm{C}$ (reversed cybotactic clusters) were observed to grow and coalesce over the collapse plateau, and phase $C$ domains within a field of phase D (cybotactic clusters) were observed upon expansion along the collapse plateau. These observations are in good agreement with the behavior associated with typical liquid crystalline cybotactic clusters. Another important result for the TPSE series is the temperature dependence found for the monolayer collapse surface pressure which gives an increase in entropy with the collapse process which is in agreement with the proposed smectic-to-nematic collapse mechanism.

As a class, the bulge amphiphiles appear to form films that exhibit all the major characteristics of liquid crystals. In contrast, the films formed by long-chain, fatty acid amphiphiles have been shown to be well-described by treating them as two-dimensional solid phases [4]. This suggests that threedimensional phase behavior can be regarded as having completely equivalent parallels in two dimensions. Of course, a liquid-crystal model describes the behavior of fatty acid Langmuir films, just as it could arguably be extended, although 
with great effort, to do the same for three-dimensional crystalline phases. The direct mapping of three-dimensional behavior into two dimensions is useful in developing two-dimensional films with interesting properties and is, at the least, of some conceptual value.

Acknowledgments - This research was supported by the Nebraska Research Initiative and the University of Nebraska Center for Materials Research and Analysis.

\section{Notes \& References}

[1] G. L. Gaines, Insoluble Monolayers at Liquid-Gas Interfaces, Interscience, New York (1966)

[2] A. Ulman, An Introduction to Ultrathin Organic Films: From Langmuir-Blodgett to Self-Assembly, Academic Press, Boston (1991).

[3] V. M. Kaganer, H. Mohwald, and P. Dutta, . Rev. Mod. Phys., 71 (1999), p. 779 and references therein.

[4] C. J. Eckhardt and T. Luty, H. S. Nalwa, Editor, Handbook of Thin Film Materials, vol. 2 Academic Press, San Diego (2002), p. 685.

[5] J. J. Haycraft, C. A. DeVries, H. Garcia Flores, A. Lech, J. P. Hagen, and C. J. Eckhardt. Thin Solid Films, 515 (2007), p. 2990.

[6] P. G. De Gennes, The Physics of Liquid Crystals, Clarendon, Oxford (1974).

[7] A. de Vries. Mol. Cryst. Liquid Cryst., 10 (1970), p. 31.

[8] A. de Vries. Mol. Cryst. Liquid Cryst., 10 (1970), p. 219.

[9] A. de Vries. Mol. Cryst. Liquid Cryst., 11 (1970), p. 361.

[10] G. Marshall, E. Teer, C. M. Knobler, M. Schalke, and M. Lösche. Coll. Surf. A: Physicochem. Eng. Aspects, 171 (2000), p. 41.

[11] The cross-sectional area of the triphenyl group was estimated as a triangle consisting of the outermost hydrogen on each phenyl ring, using the Spartan energy-minimized structure. This value gives an estimate of the area per molecule for a monolayer with close-packed triphenyl groups, $38 \AA^{2} /$ molecule.
[12] R. Casilla, W. D. Cooper, and D. D. Eley. J. Chem. Soc., Faraday Trans. 1: Phys. Chem. Condensed Phases, 69 (1973), p. 257.

[13] W. D. Harkins and L. E. Copeland. J. Chem. Phys., 10 (1942), p. 272.

[14] S. Ställberg-Stenhagen and E. Stenhagen. Nature, 156 (1945), p. 239.

[15] N. K. Adam and G. Jessop. Proc. R. Soc. Lond., A112 (1926), p. 362.

[16] J. Xue, C. S. Jung and M. W. Kim. Phys. Rev. Lett., 69 (1992), p. 474.

[17] N. R. Pallas and B. A. Pethica. Langmuir, 1 (1985), p. 509.

[18] G. M. Bommarito, W. J. Foster, P. S. Pershan, and M. L. Schlossman. J. Chem. Phys., 105 (1996), p. 5265.

[19] K. Tamada, H. Minamikawa, and M. Hato. Langmuir, 12 (1996), p. 1666.

[20] H. Sigl, G. Brink, M. Seufert, M. Schulz, G. Wegner, and E. Sackmann. Eur. Biophys. J., 25 (1997), p. 249.

[21] M. Ibn-Elhaj, H. Möhwald, M.Z. Cherkaoui, and R. Zniber. Langmuir, 14 (1998), p. 504.

[22] R. Plehnert, J.-A. Schroter, and C. Tschierske. Langmuir, 15 (1999), p. 3773.

[23] D. Honig and D. Mobius. J. Phys. Chem., 95 (1991), p. 4590

[24] S. Hénon and J. Meunier. Rev. Sci. Instrum., 62 (1991), p. 936.

[25] M. C. Friedenberg, G. G. Fuller, C. W. Frank, and C. R. Robertson. Langmuir, 10 (1994), p. 1251.

[26] A. Moore, C. M. Knobler, D. Broseta, and F. Rondelez. J. Chem. Soc., Faraday Trans., 282 (1986), p. 1753.

[27] E. K. Mann, S. Henon, D. Langevin, and J. Meunier. J. Phys. II Fr., 2 (1992), p. 1683.

[28] S. Rivière, S. Hénon, J. Meunier, D. K. Schwartz, M.-W. Tsao, and C. M. Knobler. J. Chem. Phys., 101 (1994), p. 10045.

[29] R. Rolandi, H. Schindler, M. De Rosa, and A. Gambacorta. Eur. Biophys. J., 14 (1986), p. 19.

[30] A. Sawada and S. Naemura. Jpn. J. Appl. Phys., 41 (2002), p. L195. 